anisotropy (RA), a measure of preferred directionality of perfusion (and dependent on axonal and oligodendroglial development) was $25 \%$ lower in central $\mathrm{WM}$, the site of the original injury, and $20 \%$ lower in the internal capsule that carries fibers from the injured WM. Diffusion vector maps showed alterations in the microstructure of the damaged WM and descending fiber tracts. (Huppi PS, Murphy B, Maier SE et al. Microstructural brain development after perinatal cerebral white matter injury assessed by diffusion tensor magnetic resonance imaging. Pediatrics March 2001;107:455-460). (Reprints: Joseph J Volpe MD, Department of Neurology, Children's Hospital, Fegan 1103, 300 Longwood Ave, Boston, MA 02115).

COMMENT. Perinatal cerebral white matter injury may result in both an immediate local tissue destruction and a subsequent impairment of brain development affecting fiber tracts and distal structures. These late effects of injury could reflect a Wallerian degeneration in the posterior limb of the internal capsule and a maldevelopment of axonal-oligodendroglial structures. They may explain the MRI finding of asymmetrical hypomyelination of the internal capsule and hemiplegia as sequelae of perinatal ventricular hemorrhage.

Dissociated cortical motor reorganization following brain injury. Differences in interhemispheric organization of sensory and motor pathways in cerebral palsy (CP) have been studied, using transcranial magnetic stimulation (TMS) and functional magnetic resonance imaging (fMRI), in 7 subjects with hemiplegic CP (ages 15-57 years) at the University of Western Australia and the Princess Margaret Hospital for Children, Nedlands and Subiaco, Australia. (Thickbroom GW et al. Ann Neurol March 2001;49:320-327). Normal contralateral motor and sensory projections were demonstrated between the unaffected hand and the cerebral hemisphere. Passive movement of the affected hand caused fMRI contralateral activation, whereas TMS indicated either an ipsilateral projection or asymmetrical bilateral projection. Some of the motor dysfunction in the affected hand in CP may be due to a dissociation between sensory and motor representations in cortical reorganization.

\title{
NEUROLOGIC EXAM AND MRI CORRELATIONS IN HIE
}

The correlation between scores on a standardized neurologic examination at age 9 to 14 months and early MRI findings (within 4 weeks of delivery) was determined in 53 term infants with hypoxic-ischemic encephalopathy evaluated at 2 and 4 year follow-up at Hammersmith Hospital, London, UK. Scans at 1 week after birth were normal in 16 and abnormal in 37 . Neurologic scores were optimal in 31 and suboptimal in 22. The maximum Hammersmith optimality global score for neurologic examination of cranial nerves, posture, motion, tone, and reflexes is 78 . Scores $<40$ were associated with severe disability, and between 40 and 67 reflected a restricted mobility. Lowest scores were correlated with severe basal ganglia and white matter lesions on MRI, and intermediate scores with moderate lesions. The neurologic exam was normal in 29 infants, and 35 children could walk independently at age 2 and without restrictions by age 4 year follow-up. Eight had motor restrictions and 10 showed severely limited mobility and sitting at 2 and 4 years. The magnitude of suboptimal scores on neurologic examination at approximately 1 year was related to the pattern of MRI lesions defined soon after birth and was reflective of the functional motor disability and outcome at 2 and 4 years. (Haataja L, Mercuri E, Guzzetta A et al. Neurologic examination in infants with hypoxic-ischemic encephalopathy at age 9 to 14 months: Use of optimality scores and correlation with magnetic resopnance imaging findings. I Pediatr 
March 2001;138:332-337). (Reprints: Eugenio Mercuri MD, Department of Paediatrics, Hammersmith Hospital, Du Cane Rd, London W12 0HS, UK).

COMMENT. A standardized neurologic examination at approximately 1 year of age in an infant suffering hypoxic-ischemic encephalopathy at birth is a useful measure of functional motor outcome in later infancy and early childhood. The examination at 1 year correlates with the MRI findings at or soon after birth.

Prognostic value of EEG and MRI combined in full-term infants with acute encephalopathy. The correlation between an early EEG (within 72 hours after birth) and an MRI (end of 1 week) in assessing outcome of acute neonatal encephalopathy was studied in 25 full-term infants treated at Hammersmith Hospital, London, UK. (Biagioni E, Mercuri E, Rutherford M et al. Pediatrics March 2001;107:461-468). Both EEG and MRI were predictive of the outcome at 2 year follow-up. Seven infants with normal EEG background activity and 14 with discontinuous abnormality showed normal and abnormal outcomes, respectively. Eight with normal MRI or minimal abnormalities had a normal outcome, whereas 17 with moderate to severe MRI lesions were moderately to severely impaired neurologically and 5 died.

Hypothermic therapy for hypoxic-ischemic encephalopathy. The neurodevelopmental outcome at 18 month follow-up of 40 term infants with HIE treated with head cooling and systemic hypothermia or standard normothermic care was determined at the National Women's Hospital, Auckland, New Zealand. (Battin MR et al. Pediatrics March 2001;107:480-484). The mildly cooled groups showed no worsening of outcome and a trend toward better outcome (26\% adverse outcome cf $44 \%$ among controls). The authors conclude that the short-term safety of cooling is demonstrated but the long-term efficacy is not proven. Multicenter trials are in progress.

\section{METABOLIC DISORDERS}

\section{NEUROLOGIC OUTCOME OF INFANTILE HYPOGLYCEMIA}

The neurologic development of 90 patients with persistent hyperinsulinemic hypoglycemia was studied retrospectively at Hospitalier Universitaire Necker-Enfants Malades, Paris, France. Treatment was surgical in 63 and medical in 27 . Of 54 neonates, 8 were treated medically and 46 surgically. Of 36 with infancy-onset hyperinsulinism, 19 were treated medically and 17 had pancreatectomy. Development was normal in $74 \%$. Severe mental retardation occurred in $8 \%$ of patients, and intermediate psychomotor disability in $18 \%$. Epilepsy occurred in $18 \%$ of the total (in 24\% of 54 neonates and $8 \%$ of 36 infants). The incidence of epilepsy was $7 \%$ among those with normal mental development and $57 \%$ in the severely retarded, at a mean follow-up age of 8 years. Microcephaly occurred in $10 \%$ of normal and $57 \%$ of severely retarded children. Neonatal onset was the main risk factor for severe retardation or epilepsy. Patients treated medically were less severely affected than those treated surgically. The outcome was not different in patients with diffuse and focal adenomatous hyperplasia with hyperinsulinism. (Menni F, de Lonlay P, Sevin C et al. Neurologic outcomes of 90 neonates and infants with persistent hyperinsulinemic hypoglycemia. Pediatrics March 2001;107:476-479). (Reprints: Jean-Jacques Robert MD, PhD, Diabete de l'Enfant et de l'Adolescent, Hopital Necker-Enfants Malades, 149 rue de Sevres, 75743 Paris Cedex 15, France). 